\title{
La investigación científica y su aporte al proceso de desarrollo del occidente de Honduras
}

Scientific research and its contribution to the development process of western Honduras

DOI 10.5377/rct.v0i24.7879

\author{
Jorge Humberto Orellana ${ }^{1}$ \\ María Magdalena Landaverry ${ }^{2}$ \\ Jorge Evelio Miranda ${ }^{3}$
}

\section{RESUMEN}

Este estudio presenta una valoración acerca de la pertinencia de las investigaciones científicas de las universidades públicas y privadas con presencia en el occidente de Honduras. El trabajo fue realizado entre octubre de 2016 y julio de 2017. Las herramientas utilizadas fueron principalmente cuestionarios y entrevistas con gestores del desarrollo y educación superior. Los resultados demuestran que existe una mora sustancial en investigación científica; la coordinación entre las distintas universidades para impulsar procesos de investigación y vinculación son casi nulos, existiendo un distanciamiento entre la Academia y las intervenciones del desarrollo. La investigación queda reducida a unos pocos docentes y es poco socializada. Por su parte, el desarrollo sigue percibiéndose como una meta a alcanzar y como el simple crecimiento económico. Los entrevistados perciben que las universidades deben investigar más temas relacionados con educacion, salud, ambiente, emprendimiento, seguridad alimentaria y ciudadana. Problemas que afectan a la región son el desempleo, la corrupción y la pobreza generalizada, esto se relaciona con la exigencia de la sociedad para profundizar dichos estudios. La pertinencia debe estar más en función de las necesidades de la población y no desde una visión vertical impositiva, por lo

\footnotetext{
${ }^{1}$ Profesor de la Carrera de Comercio Internacional, Coordinación Regional de Investigación Científica, Centro Regional Universitario de Occidente, CRIC-CUROC-UNAH: jorellana4000@yahoo.es

2 Profesora de la Carrera de Administración de Empresas, Coordinación Regional de Investigación Científica, Centro Regional Universitario de Occidente, CRIC-CUROC-UNAH: mlandaverry2002@yahoo.com

2 Profesor de la Carrera de Administración de Empresas, Coordinación Regional de Investigación Científica, Centro Regional Universitario de Occidente, CRIC-CUROC-UNAH: jemrouge@hotmail.com
} 
que deben definirse líneas y estudios que surjan desde la sociedad misma. Es necesaria la crítica permanente que posibiliten la búsqueda de pensamientos alternativos; deben revisarse los giros paradigmáticos de la ciencia y las intervenciones del desarrollo, esto obliga a entrar en un proceso de descolonización epistemóloga y de deconstrucción cultural que permitan el reencuentro con otros caminos.

Palabras clave: Pertinencia, investigación, universidades, desarrollo, región occidental, Honduras, pobreza.

\section{ABSTRACT}

This study presents an assessment of the relevance of scientific research in public and private universities with a presence in western Honduras. The research was conducted between October 2016 and July 2017. The tools used were mainly questionnaires and interviews with managers of development and higher education. The results show that there is a substantial delay in scientific research; The coordination between the different universities to promote research and linking processes are almost nil, and there is a distance between the Academy and development interventions. The research is reduced to a few teachers and is poorly socialized. For its part, development is still perceived as a goal to be achieved and as simple economic growth. The respondents perceive that universities should study more topics related to education, health, environment, entrepreneurship, food security and citizenship. Problems affecting the western region are unemployment, corruption and widespread poverty; this is related to the need of society to deepen these themes. Relevance must be more in line with the needs of the population and not from a vertical view of taxation, so lines and studies must be defined as emerging from society itself. It is necessary permanent criticism that make possible the search for alternative thoughts; the paradigmatic shifts of science and development interventions must be reviewed, this forces us to enter into a process of epistemological decolonization and cultural deconstruction that allow us to reconnect with other paths.

Keywords: Relevance, research, universities, development, western region, Honduras, poverty. 


\section{INTRODUCCIÓN}

Esta investigación presenta una valoración de cuál ha sido el aporte de los procesos de investigación de las diferentes universidades con presencia en la región occidental de Honduras y cómo sus hallazgos han contribuido con el desarrollo regional. Las universidades son espacios en los cuales se comparte información y conocimiento; se promueven y se analizan ideas; es un lugar para la innovación y para plantear nuevas alternativas. Desde hace varias décadas surgieron una serie de teorías desarrollistas para buscar resolver los problemas del crecimiento económico, la pobreza y exclusión social en el mundo; con diferentes enfoques han sido múltiples las intervenciones que se han realizado en nombre del desarrollo.

Qué modelo de desarrollo es posible en países como Honduras cuando dichos modelos han fracasado en el mundo. Es aquí la relevancia de las universidades con sus investigaciones científicas para aproximarse a esas realidades, dónde radica la raíz de los problemas de pobreza, vulnerabilidad y exclusión que sufre la región occidental del país, cómo se vincula ese proceso con diferentes dimensiones del llamado desarrollo. Si en el occidente de Honduras se han invertido millonarias cantidades de lempiras en proyectos de desarrollo y la región sigue tan desigual, entonces, ¿qué ha pasado?, ¿qué se ha investigado desde la Academia?, ¿cuáles son las percepciones que los gestores de la educación superior tienen del desarrollo a la hora de priorizar las líneas de investigación? Estas y otras han sido preguntas que se han reflexionado en este estudio que merecen mayores valoraciones para encontrar las causas estructurales que aquejan a la mayor parte de la población.

Las dificultades encontradas en el presente estudio es que generalmente las investigaciones científicas en las universidades no están del todo disponibles para el público. Existe una confusión entre docentes y estudiantes a la hora de aplicar metodologías de investigación puesto que muchos profesores no cuentan con la experiencia en el ramo. La obligación de investigar es casi nula y quienes lo hacen enfrentan una serie de dificultades administrativas, esto demuestra que la mora de las universidades, tanto públicas como privadas, es considerable. Se espera que este estudio contribuya a las reflexiones críticas del quehacer de las instituciones de educación superior con presencia en el occidente del país y cómo sus acciones impactan en los procesos de intervención regional.

Es imprescindible, para evitar la duplicidad de esfuerzos, la búsqueda de los espacios de coordinación entre instituciones; es necesario el estudio de las demandas más 
urgentes de la población, es imperiosa la necesidad de que las universidades trasladen la experiencia hacia lo interno de sus aulas de enseñanza, pero, además, es urgente el acercamiento de la técnica, la información y la tecnología a las personas que contribuyen con sus esfuerzos al crecimiento económico de la zona occidental. Con este trabajo, se ha intentado reflexionar para conocer y conocernos, para comprender las asimetrías de esta noble región; se trata de la búsqueda y la construcción de una nueva epistemología de intervención en la investigación y el desarrollo regional; es la búsqueda de alternativas más incluyentes y, especialmente, con rostro humano.

\section{MÉTODO}

\section{Diseño mixto: enfoque cualitativo y cuantitativo de la investigación}

Este estudio se realizó mediante técnicas de investigación cualitativa y cuantitativa. El propósito del enfoque ha sido tratar de comprender la realidad con una lógica intersubjetiva y objetiva que parte de la realidad misma, especialmente con una dinámica dialógica. El trabajo contempló una serie de entrevistas con gestores académicos y del desarrollo. La entrevista, se aplicó a técnicos y directores de organizaciones que tienen que ver con dimensiones del desarrollo como salud, educación, ambiente, emprendedurismo, seguridad ciudadana y seguridad alimentaria. Para propósitos del estudio se utilizó un "cuestionario de entrevista" el cual contempló preguntas cerradas y abiertas, este fue aplicado a la población de las cabeceras departamentales. de Copán, Ocotepeque y Lempira. Para la aplicación del cuestionario se contó con la colaboración de estudiantes de grado del Centro Regional Universitario de Occidente. El instrumento aplicado buscó recopilar información de las personas acerca del trabajo que desempeñan las Universidades en cuanto a sus investigaciones científicas y su aporte al proceso de desarrollo regional. El estudio relacionó tres variables: pertinencia, investigación y desarrollo. Durante el proceso de investigación se hicieron interpretaciones de la realidad observada, se indagaron acerca de las percepciones conceptuales que prevalecen en torno a la idea del desarrollo. Se consultaron diversas fuentes para comprender los objetivos plasmados en la investigación.

\section{Población y entorno}

El estudio fue realizado en varios municipios de la región occidental, en los cuales tienen presencia Universidades públicas y privadas; en tal sentido, las entrevistas 
fueron realizadas en Copán Ruinas, Nueva Arcadia (La Entrada), San Pedro y Corquín Copán, San Marcos y Nueva Ocotepeque en el departamento de Ocotepeque, Gracias, en el departamento de Lempira. Los cuestionarios fueron aplicados principalmente a la población de las cabeceras departamentales con un tamaño de muestra según fórmula establecida en estudios cuantitativos. Tanto las entrevistas como los cuestionarios tuvieron como propósito recopilar la percepción de actores clave acerca de cuál es la pertinencia de las investigaciones científicas y su contribución con los procesos de desarrollo regional. En promedio se realizaron entre 25 y 30 entrevistas con actores clave según las dimensiones del desarrollo priorizadas. Las entrevistas fueron realizadas principalmente en Santa Rosa de Copán, Gracias, Lempira y Ocotepeque, Ocotepeque. Para propósitos de la recopilación de la información, los cuestionarios fueron aplicados en distintos sectores de las ciudades en estudio. Los sujetos entrevistados fueron escogidos por conveniencia y por sugerencia de otros actores en la medida se avanzó con la investigación. Algunos datos numéricos procedentes de las categorías estudiadas han servido para la comprensión del tema; en ese sentido, se utilizaron 427 instrumentos/cuestionarios de los cuales el $53 \%$ se aplicó en mujeres y el $47 \%$ en hombres. La edad promedio de los consultados fue de 33 años. Con respecto a la escolaridad, el 39\% ostentaba el nivel de secundaria completa, $26 \%$ grado universitario y el resto diferentes niveles educativos. De las personas consultadas con grado universitario egresaron de la UNAH, UPNFM y UNICAH, el mayor porcentaje de egresados le corresponde a la Universidad Nacional Autónoma de Honduras. Las personas con grado universitario entrevistadas egresaron de carreras de Administración de Empresas y Ciencias Jurídicas y Sociales.

\section{Intervenciones y análisis de la información}

Para este estudio se diseñó una plantilla en SPSS que sirvió para el ordenamiento de la información cualitativa y cuantitativa procedente de los cuestionarios. Este programa sirvió para extraer categorías de análisis; es decir, percepciones conceptuales que los entrevistados fueron atribuyéndole al quehacer de las universidades en la región. Para el análisis de la información cualitativa, una vez ordenada en el programa SPSS, se utilizó el MS Word Count \& Frequency Statistics Software, este programa facilitó la búsqueda de categorías frecuentes en cada uno de los cuestionarios. Esas frecuencias sirvieron para mostrar los datos en términos porcentuales y gráfica para una mejor comprensión. Las entrevistas se grabaron en audio y se digitaron posteriormente, estas fueron aplicadas a actores clave para la investigación; destacan representantes de organizaciones como centros de salud, hospitales, ong, Ministerio Público, oficinas de la mujer, oficinas del medio ambiente, Instituto de Conserva- 
ción Forestal, Centros de Desarrollo Empresarial, entre otros. Para sustentar el análisis de la información se hizo una búsqueda exhaustiva de bibliografía, investigaciones realizadas o documentos en bibliotecas públicas y colegios de las cabeceras departamentales acerca del trabajo investigativo de las universidades, planes y estrategias de desarrollo regional.

\section{FUNDAMENTACIÓN TEÓRICA Y RESULTADOS}

\section{Marco contextual de la investigación}

La región occidental de Honduras está comprendida principalmente por los departamentos de Copán, Ocotepeque y Lempira en la cual viven aproximadamente un millón de personas. La economía del occidente el país está basada principalmente en la producción del café y en las remesas familiares. Además, en algunas ciudades existe un auge comercial por su propia dinámica y por lo que produce la cercanía con las fronteras de Guatemala y El Salvador. Otros productos que sobresalen son el tabaco, granos básicos, verduras, frutas, legumbres, caña de azúcar, ganado, madera, minería y venta de servicios. La economía del occidente de Honduras se ve obstaculizada por los costos de los insumos, la débil comercialización, la intermediación y la frágil infraestructura vial. Existen dos grupos étnicos diferenciados, chortí y lenca (Orellana, 2017). En el contexto de la región occidental existen dos universidades públicas y cuatro privadas, generalmente ubicadas en las cabeceras departamentales, sin embargo, existe presencia en la modalidad a distancia en ciudades como Copán Ruinas, San Marcos Ocotepeque, San Pedro, La Entrada y Corquín Copán, juntas reúnen no menos de cinco mil estudiantes. En términos del desarrollo, el occidente de Honduras ha contado con una serie de organizaciones no gubernamentales que reciben financiamiento de la cooperación internacional. Con diferentes enfoques y modelos, han invertido miles de millones de lempiras para buscar solventar los problemas de pobreza, aislamiento y exclusión social que sufre la región.

\section{El contexto de la investigación científica y el desarrollo del occidente de Honduras}

En el occidente de Honduras tienen presencia seis universidades; la Universidad Nacional Autónoma de Honduras, la Universidad Pedagógica Nacional Francisco Morazán, la Universidad Católica de Honduras, la Universidad Cristiana Nuevo Milenio, la Universidad Cristiana de Honduras y la Universidad Metropolitana de Honduras, de ellas, dos son públicas y el resto privadas. Ofertan diferentes carreras 
orientadas a las áreas económicas y administrativas, a las ciencias jurídicas y sociales y algunas en la rama de tecnología. La tarea de investigación en las Universidades mencionadas en el occidente de Honduras es muy limitada y en ocasiones inexistente; su labor consiste principalmente en docencia y proyectos puntuales de vinculación con la sociedad. Generalmente suele confundirse la labor de investigación con la revisión bibliográfica que se realiza a lo interno de cada asignatura. Las tareas de investigación son limitadas por diferentes razones; por una parte, existen pocos espacios para el aprendizaje de metodologías y, por otra, las pocas exigencias para cumplir con los deberes académicos de docencia, investigación y vinculación hacen que la investigación sea casi inexistente. Por lo tanto, la investigación queda reducida a un grupo de docentes interesados en investigar, principalmente pertenecientes a las universidades públicas.

Al considerar que las tareas de investigación científica son limitadas, de la misma forma, la coordinación interinstitucional y los proyectos conjuntos entre investigadores son inexistentes, esto dificulta pensar en estrategias coordinadas que beneficien los procesos de desarrollo en la región. Por consiguiente, al contar con una débil labor investigativa, difícilmente se tendrán líneas de investigación propias que surjan de la necesidad de la población del occidente de Honduras. Esto conlleva a que se dupliquen esfuerzos y presupuestos puesto que no se cuenta con mecanismos de coordinación o supervisión de las ideas de investigación emprendidas en el contexto regional. Por lo tanto, los esfuerzos de investigación científica van por un lado y los procesos y estrategias de desarrollo caminan por otro. A esta escasa coordinación e integración sumemos la tendencia común de ver el desarrollo como crecimiento económico y como una meta que debemos alcanzar; es decir, los esfuerzos de diferentes sectores públicos y privados, desde su percepción, van encaminados a convertir la región en desarrollada, con mayor infraestructura vial, edificios, tecnología y toda explotación de riqueza posible.

\section{Teorías relacionadas con el cambio de época, la innovación, el desarrollo y la investigación}

Los fundamentos filosóficos de la ciencia que se genera dentro de las universidades ha estado en profundas transformaciones y críticas. Ahora no se habla de certezas puesto que la realidad no es objetiva, si así lo fuera, existiera una sola verdad posible para explicar los fenómenos que acontecen en la sociedad; es decir, la realidad no existe independiente de la percepción humana, puesto que emerge de las verdades que cultivamos del mundo (De Souza, 2008). En tal sentido, han surgido nuevas teorías para explicar las premisas que sustentan las verdades existentes, por ejem- 
plo, la teoría del cambio de época plantea que las premisas que fueron implantadas a lo largo de los siglos ya no pueden resolverse con los mismos métodos con las cuales fueron creadas y, por lo tanto, nos induce a realizar deconstrucciones culturales que posibiliten el reencuentro con nuevos caminos. La teoría del cambio de época tuvo su génesis en la segunda mitad del siglo XX con la revolución sociocultural de los años sesenta, la revolución tecnológica de mediados de la década del setenta y la revolución económica de finales de la década del setenta.

Por su parte, la teoría de la "innovación de la innovación institucional" surgió a principios del siglo XXI liderada por la Red Nuevo Paradigma y por otras organizaciones de América Latina. Recoge una serie de teorías que han estado de la mano de la ciencia y del conocimiento: la teoría del discurso, la teoría crítica, teoría de la red de actores, teoría de la complejidad, teoría cognitiva, teoría de la acción, entre otras. Presenta una crítica a las teorías de acción como la mecanicista, economicista, evolucionista y contextual (Santamaria, 2004), las cuales se reproducen en los contextos académicos. La innovación de la innovación institucional trata de mostrarnos dos ideas para interpretar la realidad en la que vivimos, por un lado, el colonialismo y, por otro, la modernidad (De Souza, 2014); estas ideas son las que sustentan las intervenciones del desarrollo que se producen alrededor del mundo.

Por su parte, las teorías que han estado de la mano de las estrategias de intervención del desarrollo son la teoría de la modernización de los años cincuenta y sesenta del siglo XX; la teoría de la dependencia de los años sesenta y setenta y, posteriormente, desde mediados de la década del ochenta surgieron los debates pos- desarrollistas relacionados con el análisis de los discursos del desarrollo y se su práctica social (Escobar, 2005). Como estrategias de desarrollo los modernistas plantearon que las economías debían industrializarse mediante "la tecnificación de la agricultura, la urbanización, la adopción de valores occidentales, la racionalidad, el individuo, el mercado" (Estrada, 2013, p, 235). Con la modernización era posible destruir prácticas arcaicas, supersticiosas y homogeneizar la vida, "sin importar el costo social, cultural y político (Escobar, 2007, p. 78).

Por otra parte, los teóricos dependentistas plantearon que el problema de los países pobres era la expansión de los países industrializados, pero, además, la dependencia no era solo externa, sino que creaba estructuras internas en las sociedades (Blomström y Ente, 1990). Los dependentistas plantearon una serie de estrategias como la eficiente intervención del Estado, la inversión extranjera en función de las prioridades nacionales, mayor demanda interna, énfasis en políticas monetarias y fiscales y, estrategias más coherentes con el modelo de sustitución de importaciones, entre 
otros. Desde mediados de la década del ochenta se presentaron fuertes críticos a los modelos de desarrollo impulsados alrededor del mundo, los teóricos pos desarrollistas plantearon que había una subordinación de los países ricos en contra de los pobres.

Sostuvieron que el capitalismo y el patrón económico impulsado desde Estados Unidos no debía ser la categoría central que ocupara de manera total la vida de los países; es la posibilidad de crear diferentes discursos y representaciones que no se encuentren tan mediados por la construcción del desarrollo; la necesidad de cambiar las prácticas, de saber y hacer; la necesidad de multiplicar centros y agentes de producción de conocimientos; destacar las estrategias alternas producidas por movimientos sociales al encontrarse con proyectos de desarrollo (Escobar, 2005, Op. cit., p. 19). Ahora se trata de la búsqueda de pensamientos alternativas a todas las alternativas posibles, de buscar dónde radican las potencialidades; de analizar cuáles son los factores que provocan pobreza en medio de tanta riqueza. Por lo tanto, es aquí en la cual las distintas universidades tienen desafíos y retos trascendentales para contribuir con esa búsqueda que mejoren las condiciones de vida de la población.

\section{Pobreza, investigación, potencialidades y desarrollo en la región occidental de Honduras}

Reducir las desigualdades sociales en el país ha sido un reto para diferentes Gobiernos de turno. Son múltiples las observaciones de distintas instituciones tanto nacionales como internacionales acerca de la pobreza en Honduras; el Banco Mundial (BM); la Comisión Económica para América Latina (CEPAL); el Programa de las Naciones Unidades para el Desarrollo (PNUD); el Foro Social de la Deuda Externa y Desarrollo de Honduras (FOSDEH) y tantas otras, han enfatizado en las precarias condiciones en las que vive la mayor parte de la población. Según el FOSDEH (2017), en el período comprendido de 2000 a 2016 el país ha invertido en nombre de la pobreza 421 mil millones de lempiras y la población sigue tan pobre y desigual como nunca. Esta situación tiene diferentes interpretaciones; generalmente el Gobierno busca favorecer el crecimiento económico y todas sus políticas e inversiones van encaminadas a contar con la infraestructura que le permita mejorar sus índices macroeconómicos; la economía hondureña se ha basado principalmente en una economía de enclave; principalmente en comunicaciones, energía, minería, banano, maquila y sector financiero (FOSDEH, 2017). Este tipo de economía no produce un efecto derrame y no están vinculadas con otros sectores productivos, por lo que sus beneficios provocan una mayor concentración de la riqueza nacional. 
La desigualdad y el incremento de la pobreza tiene que ver, además, con la escasa redistribución de los ingresos. Honduras es un país que se sustenta en una economía basada en la mediana y pequeña empresa; de los 149,345 contribuyentes del censo empresarial del Instituto Nacional de Estadísticas INE de 2016, 127,028 contribuyentes correspondían a medianos y pequeños empresarios, únicamente 2,028 contribuyentes correspondían a la categoría de grandes empresas (FOSDEH, 2017). Según el Directorio de Establecimientos Económicos (DEE-INE), el total de empresas registradas en Copán son 8,035; en Ocotepeque 2,826 y Lempira 1,743 de las cuales en su mayoría son medianas y pequeñas empresas (DEE, 2019). En tal sentido, son los pequeños empresarios quienes financian el "desarrollo" en el país. En tal sentido, la lógica de las inversiones tendría que estar encaminada a favorecer a los sectores que más contribuyen con el fisco, sin embargo, la inversión pública ha estado orientada a la construcción de carreteras en los últimos años, lo cual significa que su impacto en el mejoramiento de las condiciones de vida de la población no es halagador.

En tal sentido, las investigaciones en las distintas universidades del país tienen un potencial para el análisis de estas realidades. Desde hace varias décadas el país no cuenta con un verdadero instrumento de planificación de corto, mediano y largo plazo. Las improvisaciones son notorias con la llegada de cada Gobierno. Si bien es cierto el país tuvo intentos de buscar sinergias e integralidad en sus estrategias de intervención a través del Plan de Nación, hace años esta iniciativa fue dejada de lado y lo que existe actualmente en el país son estrategias asistencialistas sin una verdadera vinculación con todos los sectores productivos. Falta mayor énfasis e inversión en educación, salud, fomento del emprendimiento y apoyo a sectores excluidos; es decir, es necesario cumplir con la demanda de los satisfactores de necesidades y posteriormente centrarse en cómo mejorar las libertades y la calidad de vida de las personas (Sen, 2001, p.114). Por ejemplo, en el Índice de Desarrollo Humano IDH de 2015 del PNUD Honduras se ubicó con un puntaje de 0.606, lo cual significa un empeoramiento con el 0.617 del año 2013. Este indicador que ubica a los países de acuerdo con su desempeño en el mejoramiento de la calidad de vida de sus habitantes plantea que Honduras en 2006 ocupó en el ranking internacional el puesto 108 de los 188 países analizados; en 2016 el país se ubicó en el puesto 130, lo cual representa un deterioro de vida para las personas más desposeídas (PNUD, 2016).

En tal sentido, la Academia con todo su potencial en investigación científica, podría analizar los temas del desarrollo según el contexto regional; por ejemplo, estudiar dimensiones para el disfrute de una vida larga y saludable, acceso a la educación y contar con una vida digna. Si bien es cierto Honduras ha logrado mejoras importantes al incrementarse en un 23.2\% entre 1990 y 2015 el índice de desarrollo humano, el 
país todavía continúa con rezagos en términos de participación y concentración de la riqueza. Es evidente que en términos de participación económica la región occidental de Honduras no compite con la zona norte y centro del país, sin embargo, la región occidental de Honduras cuenta con una serie de posibilidades que pueden facilitar una mejora sustancial en términos de bienestar social. Existe un potencial ecoturístico, se tiene cercanía con las fronteras de El Salvador y Guatemala. El occidente es una región rica en biodiversidad, en microclimas y en agua; existe un potencial en liderazgos y se ha fortalecido un tejido social y de coordinación entre las distintas instituciones, lo cual representa una oportunidad para mejorar sus indicadores de pobreza y de exclusión.

\section{Marco conceptual: Desarrollo, pertinencia e investigación}

El desarrollo ha sido una palabra plagada de múltiples significaciones, cada uno lo define de acuerdo con su visión de mundo prevaleciente. El desarrollo fue una invención, una tarea de superación de etapas hasta llegar a la ansiada meta del alto consumo de masas (Rostow, 1963). Un desarrollo que disfraza las ambiciones de un sistema económico globalizador que no puede estar al margen de los servicios que prestan los ecosistemas ni estar por encima de la sociedad (Max - Neef, 2006), pero que obliga a los gobernantes a crear las condiciones para tales propósitos. Ahora es difícil erradicar las concepciones tradicionales del desarrollo, puesto que el discurso ha llegado por diferentes medios, no existen países desarrollados ni subdesarrollados, sino diferentes, con diversas potencialidades, con posibilidades de crecimiento de acuerdo con los bienes propios. Para una mejor comprensión de los modelos desarrollistas, es necesario apartarse un poco de las diferentes monoculturas (Santos, 2010); es pensar en una justicia cognitiva; en la deconstrucción cultural y descolonización epistemológica, esto permitiría acercarnos más a nuestras realidades con los procesos de investigación e intervención que se realicen en la sociedad.

Por su parte, toda intervención tiene sentido en el contexto de su aplicación, por ejemplo, la pertinencia tiene que ver con el grado de acercamiento que tiene cualquier institución con la sociedad; si el fin último de todo sistema educativo es la sociedad, entonces, quién define las ofertas educativas, cómo debería involucrarse una región en el trabajo de las universidades, quién define las líneas de investigación, quién dicta las pautas para la formación de profesionales. Ante una visión mercadológica prevaleciente en la humanidad, es notorio que las universidades buscarán la formación de profesionales aptos para servir a las fuerzas del mercado y a la globalización, por lo que hablamos de competencias y de calidad antes que relevancia. La pertinencia es el involucramiento de la sociedad en la gestión de la información, en la 
comprensión de sus realidades, es traer la experiencia de la sociedad hacia los centros de educación superior; para ello, es impresionable el emprender procesos de descolonización epistemológica, no sobreponer la racionalidad económica por encima de las estrategias educativas y de desarrollo de un país.

Luis Alberto Malagón (2003) en su análisis de la pertinencia de la educación superior plantea que "la educación superior ha sido un factor necesario para garantizar el crecimiento económico en los países desarrollados y es un factor determinante para el progreso social y económico en los países en desarrollo" (pp. 118-119). Es evidente que la Universidad por si sola, no puede garantizar por medio de la pertinencia el crecimiento sostenido, ni el bienestar de la población bajo esquemas todavía coloniales de formación. Ahora es difícil tener una definición de "desarrollo", puesto que es un discurso que seduce (Rist, 2002); es pegajoso y flexible y puede ser aplicado con sus adjetivos a cualquier ámbito de la vida cotidiana, así, hemos tenido desarrollo social, económico, territorial, cultural, ecológico, participativo, comunitario, local, entre otros. En síntesis, la Universidad deberá plantearse ciertos giros paradigmáticos para una mejor comprensión de la ciencia, la educación y los procesos de investigación.

\section{Resultados del estudio}

En el occidente de Honduras tienen presencia seis universidades; dos públicas y cuatro privadas. Desde la percepción de la población consultada las más conocidas son la Universidad Nacional Autónoma de Honduras, la Universidad Pedagógica Nacional Francisco Morazán y la Universidad Católica de Honduras. Esa percepción está en función del involucramiento que tienen con los proyectos de vinculación con la sociedad y con procesos de investigación. Al consultar a la población de las cabeceras departamentales de Copán, Ocotepeque y lempira acerca de su satisfacción con el trabajo que realizan las universidades la mayoría respondió estar satisfecho, sin embargo, se pudo constatar que existe una percepción considerable de insatisfacción. Por ejemplo, las personas asocian la labor de las universidades en función de su involucramiento y en la búsqueda de solución a los problemas de la región, en tal sentido, se consultó en cuáles temas deberían realizar investigaciones, por lo que las mayores frecuencias estuvieron asociadas con temas relativos a salud, empleo, educación, seguridad y ambiente. Para cada de una de estas prioridades, desde la percepción de los entrevistados, es poco o nada el trabajo que realizan para atender estos problemas que aquejan a la sociedad.

En temas de salud, las personas recomendaron estudiar temas como el embarazo en 
adolescentes, enfermedades de transmisión sexual, la nutrición y desnutrición infantil, la protección social, medicina y atención a hospitales y centros de salud. En empleo, deben buscarse estrategias para la creación de empleos y generación de ingresos, puesto que, desde los entrevistados, los factores que condicionan las fuentes de empleos son la falta de oportunidades, el abandono del Gobierno en los departamentos, la poca inversión, el poco apoyo para programas de emprendedurismo y la creación de microempresas. En educación, deben estudiarse temas de calidad, alfabetización, estudios de mercado para oferta educativa y proporcionar desde las universidades carreras técnicas para la región. En relación con seguridad, la violencia doméstica contra la mujer y la intrafamiliar son algunos temas de investigación recomendados, igual resaltan el tema del narcotráfico y delincuencia común. En temas ambientales, se aduce que las universidades deben investigar más acerca de la contaminación que se produce en la región; la deforestación, el acceso al agua potable y estrategias para enfrentar el cambio climático.

El desarrollo durante las últimas siete décadas ha sufrido diversas críticas y modificaciones conceptuales. En esta investigación, se planteó la idea de continuar con la recopilación de información desde la gente para comprender qué se entiende por desarrollo. Al relacionar la categoría "desarrollo" con los procesos de investigación, desde la percepción de los consultados, desarrollo es empleo, educación y crecimiento económico. Su imagen más presente en la región tiene que ver con acceso a fuentes de empleo, educación e infraestructura, en tal sentido, el desarrollo se percibe como crecimiento en un sentido económico y tangible. Desde los consultados, ese desarrollo en la región se ve afectado por la falta de ingresos en las familias, la corrupción y la pobreza generalizada; es decir, se espera que el desarrollo contribuya a la calidad de vida, sin embargo, el sistema capitalista que impulsa las iniciativas de desarrollo generalmente crea un mundo invisible (Max - Neef, 2010, Santos, 2010b), por no insertarse en un mercado que exige alta productividad, sin embargo, estos sectores excluidos crean toda una red de estrategias de supervivencia que merece estudios más profundos de investigación en la región.

Para analizar de una mejor forma los procesos de desarrollo en la región y su relación con la pertenencia de las investigaciones científicas y para responder a los objetivos específicos del estudio, se escogieron seis dimensiones que tienen que ver directamente con el bienestar de la población: educación, salud, ambiente, emprendedurismo, seguridad alimentaria y seguridad ciudadana. Para la comprensión de estas dimensiones se entrevistaron a un promedio de treinta actores clave en cada dimensión seleccionada. En la dimensión de educación, la demanda de investigación para las universidades, según los entrevistados, debe ser cómo la población tiene más 
acceso a los programas educativos, esta dimensión se ve afectada por la pobreza y la desintegración familiar. La percepción es que la educación tiene una visión descendente de la realidad y a la vez monetarista, los programas educativos no están orientados a la investigación y la pobreza hace que miles de jóvenes no tengan acceso a sistemas formales de educación. La desintegración familiar fue un tema recurrente en las entrevistas; la pobreza generalizada en el occidente de Honduras hace que miles de jóvenes no encuentren fuentes de empleo y tengan que emigrar hacia otros países, principalmente, a Estados Unidos. Para el análisis de esta dimensión se entrevistaron a personas que laboran en universidades, colegios, escuelas, Supervisión Departamental de Educación y organizaciones no gubernamentales.

En la dimensión salud, los entrevistados manifestaron que los programas educativos en la educación superior relacionados con salud, no dan respuesta a los múltiples problemas y demandas que prevalecen en la región, esto se debe, en parte, a que los programas curriculares no están actualizados, falta de insumos médicos, mucha demanda y la atención es limitada; escasez de fondos asignados a salud, falta abarcar otros temas como la nutrición y, en algunos casos, existe mala atención del personal. Comentaron que deben existir investigaciones orientadas en cómo la población tiene mejor acceso a salud, a la prevención de embarazos en adolescentes y a la prevención de enfermedades como malaria y diabetes. Los embarazos en adolescentes es un tema recurrente en temas relacionados con salud, según los entrevistados, esto provoca el abandono de los estudios de cientos de jóvenes, por lo que se hace difícil su retorno e implica otros factores como la emigración hacia otros países. Las entrevistas en la dimensión salud se realizaron con personas que laboran en hospitales, clínicas públicas y privadas, centros de salud, médicos, enfermeras y organizaciones que trabajan con el tema de salud en la región.

En temas ambientales, es notorio que el occidente de Honduras presenta una serie de vulnerabilidades; la conservación de las fuentes de agua, el uso del suelo, la contaminación por las actividades productivas y la generada por los residuos sólidos y líquidos, la deforestación y las estrategias para enfrentar el cambio climático, son algunas de las preocupaciones que resaltaron en esta dimensión. Desde la visión de los entrevistados, falta más interés en temas ambientales en todo el sistema educativo, de la misma forma, debe estudiarse cuáles son las implicaciones ambientales de los procesos de desarrollo. De las sugerencias recibidas en las distintas entrevistas, se manifiesta que el problema del impacto ambiental deber ser una prioridad para la región occidental, puesto que ha sido una de las zonas más vulnerables al cambio climático. Los ciudadanos deben aprender ahora a tener mayor resiliencia en todos los sentidos. El problema ambiental no debe verse únicamente como un tema de 
contaminación y de daño a los ecosistemas, debe analizarse con toda la integralidad y no obviar las variables económicas que han dejado históricamente de lado a la madre Tierra. Para los propósitos del análisis de esta dimensión se entrevistaron a personas que laboran en las Unidades Ambientales Municipales, Instituto de Conservación Forestal, Comité Permanente de Contingencias y algunas organizaciones no gubernamentales como APDI, ASIDE, COLOSUCA, ASONOG, ATRIDEST, Plan Trifinio y Mancomunidad Trinacional.

La dimensión de emprendimiento se analizó con información procedente de actores que impulsan en la región iniciativas empresariales como las oficinas de la mujer de las municipalidades y algunas organizaciones con presencia en la región como el Centro de Desarrollo Empresarial CDE, Asociación de Organismos no Gubernamentales ASONOG y la Agencia para el Desarrollo Estratégico Local de Santa Rosa de Copán ADELSAR. De los entrevistados, la mayoría consideran que los programas de educación superior no se ajustan a la realidad regional; existe poca ayuda en términos de información y financiamiento y los programas emprendidos no tienen el debido seguimiento o acompañamiento, por lo que el emprendedor se ve sólo ante los múltiples problemas que enfrenta como pequeño empresario. Para resolver estas dificultades, las universidades con presencia en la región deberían enfocar sus esfuerzos de investigación en cuál es el impacto económico de la generación de empleo desde los emprendedores, análisis de necesidades reales con enfoques de emprendimiento, turismo con orientación en el emprendimiento, investigación de mercados, potencialidades para diversificar los negocios y cubrir necesidades empresariales.

Con relación a la dimensión de seguridad alimentaria se puede constatar que el problema en el occidente de Honduras no es la falta de alimentos; es un problema de acceso desigual a los bienes que sustentan la vida. El occidente de Honduras cuenta con las condiciones climáticas propicias para suplir de alimentos a toda su población, sin embargo, la tenencia de la tierra, la pobreza generalizada, la desigual distribución de ayudas y riqueza, hacen que la gente sufra el embate de los problemas relacionados con su alimentación. Por tal razón, desde la percepción de los entrevistados, la Universidad debe enfocar sus esfuerzos en estudiar mejor el tema de la seguridad alimentaria en la región, a pesar de que existen otras prioridades en investigación como salud, seguridad, pobreza, contaminación, desempleo; es necesario el estudio de aquellos productos que afectan la alimentación de la población, por ejemplo, el uso de productos transgénicos, la influencia de los agroquímicos en la salud y alimentación, la desnutrición y el uso de semillas criollas, la alternativa de la producción orgánica, entre otros. Para el análisis de esta dimensión se visitaron actores clave como la Mancomunidad Trinacional, ATRIDEST, Programa Mundial de Alimentos, 
oficinas de Gobierno, hospitales, centros de salud, y otras instituciones relacionadas con la seguridad alimentaria. La seguridad ciudadana es una de las dimensiones más visibles en las estrategias de desarrollo del país, sin embargo, existen temas en investigación relacionados con esta área que deben tener más coherencia con el contexto de su aplicación. Los resultados demuestran que existen temas que ni siquiera se han intervenido a nivel de exploración, para el caso, no existen organismos que se dediquen a monitorear la mora judicial, el mapeo de las zonas en conflicto, la cultura de la denuncia, los factores detonantes de la violencia y la verdadera percepción de los pobladores relacionado con la paranoia en la convivencia ciudadana. Se realizaron entrevistas con abogados de la Corte Suprema de Justicia, bufetes y Juzgados de Letras en la región, Ministerio Público y Policía Nacional. Más de la mitad de los entrevistados reconocen que no hay un vínculo entre sus instituciones y la Academia, es aquí en donde surge la inquietud, entonces, la educación superior qué es lo que enseña, para quién y de qué forma. Según se pudo constatar, algunos temas como violencia doméstica, robos, zonas de riesgo ciudadano, drogas, maras y pandillas, narcotráfico y movilidad ciudadana, han sido investigados de manera superficial, puesto que las investigaciones que se produjeron no contemplaron la rigurosidad científica, sino que respondieron a un sondeo bibliográfico o prueba piloto exigido en algunas asignaturas como requisito del pensum académico.

Al analizar cada una de estas dimensiones que están inmersas en el "desarrollo" de la región occidental, se puede constatar que existe una mora sustancial en términos de investigación científica, no se cuenta con espacios para la coordinación y la promoción de la investigación entre universidades. En algunas de ellas, las investigaciones realizadas no han encontrado los mecanismos idóneos para la socialización de sus resultados. Las tareas de investigación, en la mayoría de los casos, no es una prioridad institucional, puesto que es realizada por un grupo reducido de docentes. $A$ pesar de ello, con respecto a sus labores de investigación, las universidades mejor evaluadas por los entrevistados en la región occidental son la Universidad Nacional Autónoma de Honduras, la Universidad Pedagógica Nacional Francisco Morazán y la Universidad Católica de Honduras respectivamente.

\section{DISCUSIÓN}

La pertinencia es la capacidad que tienen las universidades con sus procesos de vinculación e investigación de responder a los problemas que aquejan a la sociedad. Son múltiples los debates acerca del nuevo rol de la ciencia, de la problematización y 
del involucramiento directo de las universidades en el contexto de sus realidades. Es la sociedad quien debe dictar las pautas para que las universidades a través de sus investigaciones se involucren en la búsqueda de soluciones a las demandas de la población; es decir, debe ser la ciencia de la sociedad y no la ciencia para la sociedad. Durante este trabajo, según se pudo constatar en las diferentes entrevistas e instrumentos aplicados, la sociedad espera que las universidades se involucren más en los problemas regionales, que sus ofertas académicas estén más apegadas a una realidad contextual. Generalmente existe una lógica vertical de arriba hacia abajo en los procesos de intervención, tanto en temas del desarrollo como en las investigaciones científicas. Las líneas de investigación de los centros regionales son definidas en las sedes centrales, no existe, por lo tanto, una política universitaria para definir líneas de investigación regional. En su defecto, son los investigadores quienes deciden qué investigar; son los investigadores quienes siguen líneas ya establecidas de antemano. En otro sentido, dentro de las universidades no existen verdaderas exigencias para sus docentes en el involucramiento directo con la sociedad, la labor investigativa queda reducida a un pequeño grupo de profesores; en algunas universidades privadas la no exigencia radica en el hecho de que la mayoría de sus docentes son contratados únicamente por hora y se limitan a la labor docente.

En función de la pertinencia y ciertas dimensiones del desarrollo, al contar con escasos espacios para el debate dentro de las universidades dificulta la reflexión de cuáles deberían ser las estrategias de intervención en la región. No se debate acerca de los nuevos cuestionamientos a los modelos de desarrollo, no se debate acerca de las críticas a la ciencia moderna. Los procesos formativos en metodologías de investigación son limitados y quienes acceden a ellos, lo hacen no necesariamente como un deber manifiesto a lo interno de la institución. Esta situación es desfavorable para la pertinencia en los procesos de investigación, la escasa socialización de resultados y la inexistente coordinación en tareas de investigación entre las distintas universidades dificulta aún más fortalecer la ciencia en el contexto regional. Por ejemplo, para la mayoría de las personas el desarrollo es visto como crecimiento económico. Según se constató, basta crecer para satisfacer las necesidades humanas. Durante este trabajo se pudo verificar que las investigaciones científicas no responden a una programación establecida, generalmente surgen de la espontaneidad. En aquellos centros regionales con sus oficinas centrales en otras ciudades, los temas de investigación ya vienen establecidos según la programación académica; es decir, desde otros contextos se les dice qué investigar. En algunas cátedras se plantean ideas que se plasman en un perfil de proyecto. Se pudo constatar la falta de mecanismos de coordinación entre universidades para divulgar su quehacer investigativo. Aquellas investigaciones que se han realizado en las distintas universidades generalmente no 
están disponibles al público, al no contar con una biblioteca de forma ordenada para estos trabajos sus resultados permanecen invisibles, esto conduce inevitablemente a la duplicidad de esfuerzos en tareas de investigación y aprendizaje.

En algunas cátedras de grado relacionadas con investigación no necesariamente llevan consigo una rigurosidad metodológica apegadas al llamado "método científico", esto conduce a que los estudiantes que posteriormente ingresan a posgrados lleven consigo verdaderas limitaciones. Los espacios académicos deben servir para la crítica y el debate, para la reflexión y para la innovación. Ahora enfrentamos un cambio de época que exige innovar la forma de innovar; es necesario dar ciertos giros paradigmáticos y poner en la mesa de discusión las falsas premisas y monoculturas que condicionan nuestra forma de pensar. Esta investigación recalca que es necesario replantear la forma de hacer investigación en la región, negociar la pertinencia en función de las demandas de la sociedad, sistematizar y aprovechar la experiencia, buscar mecanismos de coordinación y socialización de los resultados de investigación, esto posibilitará que las nuevas investigaciones sean más pertinentes a los problemas que sufre la región occidental; es vital que existan posicionamientos desde la Academia para la vulnerabilidad, el cambio climático, la desnutrición, el hambre, la pobreza y la exclusión que sufren miles de personas en esta parte del país.

\section{CONCLUSIONES}

Este estudio demuestra que existe una mora significativa en términos de investigación científica en la región occidental de Honduras. Las universidades en esta parte del país generalmente asumen procesos impuestos desde sus sedes centrales, con una lógica vertical y desconociendo el contexto regional. Las ideas y demandas de investigación corresponden a la iniciativa del docente y no resultan de las necesidades sentidas por la población. En el caso de la UNAH, al no contar con protocolos, procedimientos y estrategias de valoración y exigencias del desempeño laboral en materia de investigación, provoca, en cierto modo, un ambiente poco motivador para los investigadores; es decir, los docentes que están dedicados y comprometidos con los quehaceres investigativos se ven condicionados por la falta de apoyo administrativo y las escasas o nulas condiciones para la realización de estudios que requieran la disponibilidad de más de una hora de reasignación académica. Esto obliga a revisar cuáles podrían ser los mejores incentivos para esta labor que requiere disciplina, dedicación y más horas laborales para su cumplimiento. 
Durante este estudio se pudo constatar que existen al menos cuatro formas de llevar a cabo procesos de investigación. En primer lugar, existen investigaciones producto de formación de posgrados, generalmente, maestrías. En algunas universidades este tipo de investigaciones más es un estudio de factibilidad que una investigación que compruebe hipótesis, formule categorías de análisis o que resuelva un problema en particular. En segundo lugar, están las investigaciones que realizan los docentes con rigurosidad científica y metodológica en diversos temas; estos están asociadas a convocatorias de becas de investigación, estas permiten presentar ensayos o artículos científicos, los cuales son publicados en revistas científicas del país. En tercer lugar, se encuentran las investigaciones que se estipulan en los pensum de algunas carreras, pero que se limitan a elaborar un perfil de investigación. En cuarto lugar, se ubican aquellas "investigaciones" que se realizan a lo interno de las asignaturas. Este tipo de asignaciones suele confundirse al usar la palabra "investigación", puesto que no llevan una rigurosidad metodológica ni se aproximan a normas establecidas para los procesos de investigación, las mismas se limitan a una revisión bibliográfica en la cual el estudiante analiza un tema en particular y comparte los hallazgos teóricos encontrados.

La pertinencia de las investigaciones en el occidente de Honduras es cuestionable, es difícil deducir cuál ha sido el impacto durante la última década en términos de su contribución al desarrollo. La poca socialización de resultados de investigación, los escasos mecanismos de coordinación y la inexistencia de políticas regionales de investigación dificulta la comprensión de los impactos del quehacer investigativo en relación con el desarrollo regional. Por ejemplo, en educación la prioridad de investigación, según sugerencias de los entrevistados, está en estudiar las competencias y potenciar la educación superior, así como la educación especial. En la dimensión salud, las demandas estuvieron orientadas a cómo mejorar el acceso a la salud, a la prevención de embarazos en adolescentes y a la prevención de enfermedades como malaria y diabetes. En ambiente, las demandas de investigación se orientaron a la conservación de las fuentes de agua, contaminación, impacto y mitigación del cambio climático; estudios de suelo y manejo del bosque. En la dimensión de emprendimiento, los esfuerzos deben enfocarse en analizar el impacto económico que genera el emprendimiento en la región; empleo, turismo, potencialidades y necesidades de emprendimiento. En la dimensión seguridad alimentaria, la demanda está en cómo tener acceso a los alimentos y, por otra parte, orientar los estudios en función del análisis de producción transgénica y sus impactos en la salud, economía y cultura de la región. En temas de seguridad ciudadana, se demanda estudios de investigación que tengan que ver con la prevención; delincuencia, focos o vectores de violencia regional, drogas, robos, entre otros. En temas del desarrollo, este suele confundirse 
con el crecimiento económico; es decir, desde la percepción de los entrevistados una ciudad está más desarrollada en la medida que existe más infraestructura y existe más comercio. Desarrollo es empleo, educación y crecimiento económico. Las categorías que han resaltado para comprender el desarrollo son la tecnología, la infraestructura, riqueza, evolución, progreso y calidad de vida.

La investigación presenta un reto en sí misma, por ejemplo, la coordinación entre las distintas universidades es casi nula. Es imprescindible contar con un centro o repositorio de los estudios que se realizan en el contexto regional, esto podría visibilizar más el quehacer investigativo. Es imprescindible los procesos de formación en metodologías de investigación y, quienes imparten estas cátedras en las universidades, deberían contar con la experiencia necesaria que fortalezca esta labor. Deben existir espacios para debatir los nuevos retos de la ciencia, pues aún somos rehenes de premisas que culturalmente condicionan nuestra forma de ser, pensar y sentir. Las cátedras están llenas de un lenguaje epistemológicamente colonial. Aún se cree tácitamente en que la investigación debe ser objetiva para que sea científica y, por tanto, que tenga validez, sus hallazgos deben ser medibles y traducidos al lenguaje matemático; debe ser neutral y debe separarse el sujeto del objeto; es decir, existe una visión universalista, mecanicista, racionalista, reduccionista y determinista de la realidad. En este sentido, si bien es cierto la valoración que le dan a las universidades es buena, no es menos cierto, que existen muchas críticas al respecto. Las universidades, desde la percepción de los entrevistados, forman profesionales para un "mundo que no existe"; son personas que salen como fuerza laboral para el mercado, pero no encuentran las oportunidades de trabajo, por lo tanto, según lo manifestado, miles de jóvenes se ven en la obligación de emplearse en aquello para lo que no fueron formados y los obliga a procesos de emigración fuera del país.

\section{AGRADECIMIENTOS}

Agradecemos a la Dirección de Investigación Científica de la Universidad Nacional Autónoma de Honduras por permitirnos fortalecer nuestras capacidades en los temas de investigación. A las autoridades del Centro Regional Universitario de Occidente por toda su colaboración en el trabajo realizado. A los estudiantes del CUROC quienes contribuyeron con la recopilación de información. A todos, muchas gracias. 


\section{REFERENCIAS BIBLIOGRÁFICAS}

Blomström, M. y Ente, B. (1990). La teoría del desarrollo en transición. México DF: Fondo de Cultura Económica.

Directorio de Establecimientos Económicos DEE-INE (2019). Contribuyentes. Mediana y Pequeña Empresa. Tegucigalpa, Honduras. Recuperado de: http://170.238.108.227/binhnd/RpWebEngine.exe/Portal?BASE=DEE1\&lang=ESP

De Souza Silva, J. (2014). La crisis global de la "innovación para el desarrollo". Del positivismo al constructivismo para construir modos de vida localmente sostenibles. Revista Cubana de Ciencia Agrícola, (1), 48, 11-15.

De Souza, Silva, J. (2008). Otro paradigma para el desarrollo humano sustentable. Ascenso y declinación de la "idea de desarrollo". Revista de la Pontifica Universidad Católica del Ecuador, 85, pp. 25-46.

Estrada, M. (2013). Postdesarrollo, Modernidad y Otros Mundos. Entrevista con Arturo Escobar. OXÍMORA REVISTA INTERNACIONAL DE ÉTICA Y POLÍTICA, (2), 233-248.

Escobar, A. (2007). La Invención del Tercer Mundo: Construcción y reconstrucción del desarrollo. Caracas, Venezuela: Fundación Editorial el perro y la rana.

FOSDEH (2017). Perspectivas económicas 2017. Una mirada a la economía hondureña. Tegucigalpa, Honduras: USAID/IMPACTOS/COSUDE/Iniciativa Think Tank.

Hernández, Á. (2007). El Desarrollo como problema ¿lgualdad de qué? Caracas, Venezuela: UCV.

Malagón, L. A. (Julio - Septiembre, 2003). La Pertinencia en la Educación Superior: Elementos para su Comprensión. Revista de la Educación Superior, 32, (127), 113 - 134.

Max - Neef, M., Elizalde, A. \& Hopenhayn, M. (2010). Desarrollo a escala humana. Opciones para el futuro. Madrid, España: Biblioteca CF+S.

Max - Neef, M. (2006). El poder en la globalización. Revista Futuros, IV, (14), 1 - 11.

Orellana, J. (2017). Cultura silenciosa. Tegucigalpa, Honduras: Editorial Universitaria.

PNUD (2016). Informe sobre Desarrollo Humano Honduras 2016. Desarrollo humano para todas las personas. Canadá: Lowe - Martin Group.

Rist, G. (2002). El desarrollo: historia de una creencia occidental. Madrid, España: Los libros de la Catarata.

Rostow, W. (1963). Las etapas del crecimiento económico: un manifiesto no comunista. México: Fondo de Cultura Económica.

Santos, B. (2010). Para Descolonizar Occidente: Más Allá del Pensamiento Abismal, Buenos Aires, Argentina: CLACSO.

Santos, B. (2010b). La hora de los invisibles. En I. León (cood.). Sumak Kawsay / Buen Vivir y cambios civilizatorios. (2da. Edición). Quito, Ecuador: FEDAEPS.

Santamaría, J. (2004). Teorías de acción para la innovación institucional. R\&D Organizations. ISNAR Briefing Paper, 74.

Strauss A., y Corbin, J. (2002). Bases de la investigación cualitativa. Técnicas y procedimientos para desarrollar la teoría fundamentada. Medellín, Colombia: Editorial Universidad 
de Antioquia.

Sen, A. (2001). El Nivel de Vida. Editorial Complutense: Madrid, España. 